\title{
Validity of the Color Dipole Approximation for Diffractive Production of Heavy Quarkonium
}

\author{
K. Suzuki ${ }^{1 *}$, A. Hayashigaki ${ }^{1 \dagger}$, K. Itakura ${ }^{2 \ddagger}$, J. Alam $^{1 \S}$, T. Hatsuda ${ }^{1 * *}$ \\ ${ }^{1}$ Department of Physics, University of Tokyo, Tokyo 113-0033, Japan \\ ${ }^{2}$ Radiation laboratory, RIKEN, Wako, Saitama 351-0198, Japan
}

\begin{abstract}
We study the diffractive photo- and leptoproductions of $J / \psi$ and $\psi^{\prime}$ on the proton, and examine the validity of the small-size color dipole approximation to the production of radially excited heavy quarkonium. The dipole model predicts a small ratio of $\psi^{\prime}$ to $J / \psi$ photoproduction cross sections, which does not agree with experimental data. We show that this discrepancy originates from a large transverse size of $\psi^{\prime}$ which makes the convergence of the transverse size expansion questionable, and the calculation without the dipole approximation turns out to be consistent with the data. Productions of $\Upsilon^{\prime}(2 S, 3 S)$ are also discussed, and the dipole approximation is found to be reasonable for the $\Upsilon$-family.
\end{abstract}

13.85.Ni,14.40.Gx,12.38.Bx,12.39.Jh

Typeset using REVTEX

\footnotetext{
*e-mail: ksuzuki@nt.phys.s.u-tokyo.ac.jp

†e-mail: arata@nt.phys.s.u-tokyo.ac.jp

‡e-mail: itakura@rcnp.osaka-u.ac.jp

$\S$ e-mail: alam@nt.phys.s.u-tokyo.ac.jp

**e-mail: hatsuda@phys.s.u-tokyo.ac.jp
} 


\section{INTRODUCTION}

Recently diffractive phenomena in QCD have attracted considerable theoretical and experimental interests. In particular, diffractive photo- and leptoproductions of vector mesons off the proton, $\gamma^{(*)}+p \rightarrow V\left(\rho^{0}, \omega, \phi, J / \psi, \ldots\right)+p$, provide crucial constraints on properties of the QCD Pomeron and the vector meson wave functions [1] 8]. In this report, we focus on the heavy quarkonium production, where the heavy quark mass plays a role of the hard scale. Due to the Regge kinematics, the vector meson production can be understood as the scattering of the color dipole with the target nucleon. In this case we expect the amplitude to be dominated by the four diagrams illustrated in Fig.1. The upper part of each diagram, where the heavy quarks interact with two-gluons, is assumed to be calculated within perturbative QCD even for the photoproduction. On the other hand, the lower blob represents both non-perturbative structure (impact factor) of the proton and complicated gluon dynamics at small- $x$ which is identified with the QCD Pomeron.

The color dipole approach is adopted to evaluate the $J / \psi$ production in refs. [2, 33,6], and gives reasonable results in agreement with the data. However, as pointed out by Hoyer and Peigné [9] very recently, the ratio of $\psi^{\prime}$ to $J / \psi$ photoproduction cross sections calculated in the dipole approximation is much smaller than the measured values [10] at HERA, when one uses typical non-relativistic quark models to obtain the charmonium wave functions. The formula used in the dipole approach [2, 3, 9] is obtained by assuming that both transverse momentum of exchanged gluons and the transverse size of the $c \bar{c}$ pair are small. However, the non-relativistic quark model indicates that the typical size of $\psi^{\prime} \sim 0.8 \mathrm{fm}$ is twice as large as the radius of $J / \psi$. Hence, the validity of the small-size dipole approximation is questionable for $\psi^{\prime}(2 S)$ and other mesons with the large radii. We may expect considerable contributions from the scattering of the color dipole with a large transverse fluctuation, if the $\gamma$ and $c \bar{c}$ wave functions allow such a large separation between the quarks. Above argument motivates us to calculate the diffractive $J / \psi$ and $\psi^{\prime}$ productions without resorting to the dipole approximation. 


\section{CALCULATION OF DIFFRACTIVE HEAVY QUARKONIUM PRODUCTION}

Let us briefly introduce the framework to calculate the diffractive heavy quarkonium production, which was developed in ref. [3]. We consider the forward heavy vector meson production $\gamma^{(*)}(q)+p(p) \rightarrow V(q)+p(p)$ shown in Fig.1 with the kinematical condition $W^{2} \equiv(p+q)^{2} \gg Q^{2}\left(=-q^{2}\right)$. In this small- $x$ kinematics, the lifetime of a color-singlet quark-antiquark fluctuation is large compared to the typical interaction time scale [11]. Then the production amplitude can be expressed as a convolution of the $q \bar{q}$-proton cross section with the wave functions of initial $(\gamma)$ and final $(V)$ states. In terms of light-cone wave functions of the photon $\psi_{\gamma}$ and vector meson $\psi_{V}$, the production amplitude at $t=0$ is proportional to

$$
\mathcal{A}(z)=4 \pi \alpha_{s} \int d^{2} b \int d l_{t}^{2} \frac{f\left(x, l_{t}\right)}{l_{t}^{4}} \psi_{\gamma}\left(Q^{2} ; z, b\right)^{*}\left[1-J_{0}\left(l_{t} b\right)\right] \psi_{V}(z, b)
$$

where $z$ and $b$ are longitudinal momentum fraction of a quark and transverse distance between the $c \bar{c}$ pair, respectively. $x$ is the longitudinal momentum fraction of the gluon $x \approx\left(Q^{2}+4 m^{2}\right) / W^{2}$ with $m$ being the heavy quark mass, and $l_{t}$ the gluon's transverse

momentum. $f\left(x, l_{t}\right)$ denotes the unintegrated gluon number density of the proton, which contains a complicated non-perturbative gluon ladder. $\alpha_{s}$ and $J_{0}\left(b l_{t}\right)$ represent the QCD coupling constant and the ordinary Bessel function of the first kind. After multiplying suitable functions of $z$ and kinematical factors, and carrying out $z$-integration, we obtain both longitudinal and transverse differential cross sections for the heavy quarkonium production [3];

$$
\left(\frac{d \sigma}{d t}\right)_{t=0} \propto\left[\frac{Q^{2}}{M_{V}^{2}}\left(\int_{0}^{1} d z z(1-z) \mathcal{A}(z)\right)^{2}+\left(\frac{m^{2}}{4 M_{V}^{2}} \int_{0}^{1} \frac{d z}{z(1-z)} \mathcal{A}(z)\right)^{2}\right]
$$

where $M_{V}$ is the mass of the heavy quarkonium.

In eq. (11) the photon wave function $\psi_{\gamma}$ with 4 -momentum square $Q^{2}$ is calculated perturbatively, while $\psi_{V}$ is a non-perturbative object. We shall use the non-relativistic potential model which successfully reproduces the properties of the heavy quarkonium to evaluate $\psi_{V}$. 
In the following calculations, we use two types of the potential; the QCD motivated potential which essentially consists of linear and Coulomb potentials [12], and a phenomenological logarithmic potential [13]. Our procedure to evaluate the light-cone wave functions is in line with those of refs. [3.9]. Solving the Schrödinger equation with a potential $V(r)$, we obtain non-relativistic wave functions $\psi_{V}(\vec{r})$, and also subsequently those in the momentum space $\psi_{V}(\vec{k})$ through the Fourier transformation. We make a simple kinematical identification between light-cone variables $\left(z, k_{t}\right)$ and $\vec{k}=\left(k_{z}, \vec{k}_{t}\right)$ as

$$
z=\frac{1}{2}\left(1+\frac{k_{z}}{\sqrt{k^{2}+m^{2}}}\right)
$$

where $\pm k_{t}$ are the transverse momenta of the quarks. This relation gives the light-cone wave function;

$$
\psi_{V}\left(z, k_{t}\right)=\left(\frac{k_{t}^{2}+m^{2}}{4[z(1-z)]^{3}}\right)^{1 / 4} \cdot \psi_{V}\left(|\vec{k}|=\sqrt{\frac{k_{t}^{2}+(2 z-1)^{2} m^{2}}{4 z(1-z)}}\right) .
$$

Finally, performing the two-dimensional Fourier transformation in the $k_{t}$ space, we obtain the light-cone wave function $\psi_{V}(z, b)$.

If we take the infinitely heavy quark mass limit, where the Fermi motion of heavy quarks in the quarkonium can be entirely neglected, i.e. $\psi_{V}=\delta(z-1 / 2) \delta^{(2)}(b)$, the production cross section is simply related to the leptonic decay width of the heavy quarkonium [1]. However, for the charmonium, there exist substantial Fermi motion corrections at small $Q^{2}$ [3, 4, 6].

Assuming that $\left(b l_{t}\right) \ll 1$ and keeping terms up to second order of $\left(b l_{t}\right)$ [3, 6, 9], one can rewrite eq. (1) as

$$
\mathcal{A}(z)=\alpha_{s} x G\left(x, Q_{e f f}^{2}\right)\left[\pi \int d^{2} b \psi_{\gamma}\left(Q^{2} ; z, b\right)^{*} b^{2} \psi_{V}(z, b)\right]
$$

where $Q_{\text {eff }}^{2}=z(1-z) Q^{2}+m^{2}$. In eq. (5) the unintegrated gluon density has been identified with the gluon distribution function measured in DIS via [11]

$$
f\left(x, l_{t}\right) \equiv l_{t}^{2} \frac{\partial x G\left(x, l_{t}^{2}\right)}{\partial l_{t}^{2}}
$$


The approximation (5) is often called the color dipole approximation. It gives a simple physical interpretation of this process, in which the scattering amplitude of the $q \bar{q}$ dipole with the target has a geometrical expression, $\sigma_{q \bar{q}} \propto b^{2}$. In addition, the factorized formula eq. (5) makes it possible to constrain the gluon distribution function of the proton from the diffractive vector meson production. Typical results for $J / \psi$ production cross section obtained by using eq. (5) are available in refs. 3,14, and in agreement with the data. However, if we focus on $\psi^{\prime}$, which is a radially excited $2 S$-state of the charmonium, the calculated ratio of $\psi^{\prime}(2 S)$ to $J / \psi(1 S)$ cross sections at the photoproduction point $Q^{2}=0$ is much smaller than the experimental data as pointed out in [9]. The ratio calculated using eq. (5) is shown by the dashed curve in Fig.2. Although the theoretical prediction agrees with the data at larger $Q^{2}$, it has a factor 3 discrepancy at $Q^{2}=0$. We have tried to evaluate it with several potential models, but none of them can describe the data as far as the parameters of the potential are fixed to reproduce the charmonium properties [14].

Let us now examine carefully the dipole approximation which leads us to eq. (5). For the leptoproduction with large $Q^{2}$ eq. (5) works well, since the photon wave function projects out the $c \bar{c}$ fluctuation with the small transverse size. This approximation is also reasonable for the photoproduction of the $1 S$ heavy quarkonium due to the small transverse size of the $J / \psi$. However, for the photoproduction of the radially excited vector mesons, it is not clear whether the small- $\left(b l_{t}\right)$ expansion of eq. (5) is valid. Since the size of $\psi^{\prime}(2 S)$ turns out to be about $0.8 \mathrm{fm}$, twice as large as that of $J / \psi(1 S)$, in the typical non-relativistic quark model, the color dipole expansion may not work in this case. Namely, there may exist non-negligible contributions to the integral eq. (11) ¿from an overlap of the large-size $c \bar{c}$ configuration and the $\psi^{\prime}$ wave function.

In this work we explicitly evaluate eq. (11) instead of using the simplified formula (5). To do this, we need the explicit form of the unintegrated gluon density $f\left(x, l_{t}\right)$, which is not precisely known. Here, we follow the prescription of refs. [15, 4] to carry out the $l_{t}$-integration in eq. (II) by relating $f\left(x, l_{t}\right)$ to the derivative of the gluon distribution; 


$$
\begin{aligned}
\int_{0}^{Q_{e f f}^{2}} d l_{t}^{2} \frac{f\left(x, l_{t}\right)}{l_{t}^{2}} A\left(l_{t}\right) & =\int_{0}^{Q_{0}^{2}} d l_{t}^{2} \frac{f\left(x, l_{t}\right)}{l_{t}^{2}} A\left(l_{t}\right)+\int_{Q_{0}^{2}}^{Q_{e f f}^{2}} d l_{t}^{2} \frac{f\left(x, l_{t}\right)}{l_{t}^{2}} A\left(l_{t}\right) \\
& \sim x G\left(x, Q_{0}^{2}\right) A\left(l_{t}=0\right)+\int_{Q_{0}^{2}}^{Q_{e f f}^{2}} d l_{t}^{2} \frac{\partial x G\left(x, l_{t}^{2}\right)}{\partial l_{t}^{2}} A\left(l_{t}\right)
\end{aligned}
$$

where $A\left(l_{t}\right)$ is a given function of $l_{t}$ in eq. (1) , and $Q_{0}^{2}$ plays a role of the infrared separation scale.

\section{RESULTS AND DISCUSSIONS}

Using the CTEQ4LQ [16] parametrization for the gluon distribution and taking $Q_{0}^{2}=$ $0.8 \mathrm{GeV}^{2}$, we obtain the result shown in Fig.2. Calculation without (with) the dipole approximation is depicted by the solid (dashed) curve. For large $Q^{2}$ the difference between them

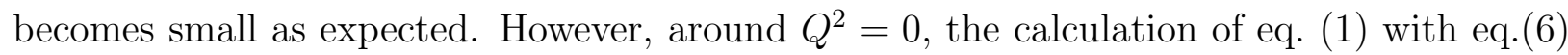
clearly larger than the result obtained by eq. (5) by factor about 2 [17], and shows a reasonable agreement with the experimental data [10]. We demonstrate the major improvement of the ratio is caused by the contribution from the large-size $q \bar{q}$ fluctuation. There may be several other mechanisms to enhance the $\psi^{\prime}$ to $J / \psi$ ratio further, which will be discussed elsewhere [14].

If we vary the value of $Q_{0}^{2}$ from $0.4 \mathrm{GeV}^{2}$ to $1 \mathrm{GeV}^{2}$, resulting ratio changes within a few $\%$. Our results are also found to be insensitive to the choice of the potential model. We show in Fig.3 results with GRV98LO [18] parametrization for the gluon distribution function (thinsolid curve) in comparison with those with the CTEQ4LQ distribution (thick-solid curve). Calculated ratio is slightly modified due to the difference of the gluon distributions between CTEQ4 and GRV98 at low $Q^{2}$.

For completeness, sensitivity of the results to $Q_{e f f}^{2}$ is demonstrated in Fig.3, because several choices of $Q_{\text {eff }}^{2}$ are adopted in the literature [3,4:6]. In Fig.3 results with $Q_{\text {eff }}^{2} \rightarrow \infty$ as an extreme case are shown by the thick-dashed (CTEQ) and thin-dashed (GRV) curves, while those with $Q_{e f f}^{2}=Q^{2} / 4+m^{2}$ by the solid curves. Although increasing the upper limit of the $l_{t}$-integration improves the result, significant enhancement at $Q^{2}=0$ comes from 
higher order terms of $b^{2}$ in eq. (四).

We also consider the production of $\Upsilon(1 S, 2 S, 3 S)$-states. One can naively expect that the transverse separation $b$ is small enough to ensure the validity of the dipole expansion for the bottom quark case. Results for $\Upsilon^{\prime}(2 S) / \Upsilon(1 S)$ and $\Upsilon^{\prime}(3 S) / \Upsilon(1 S)$ are shown in Fig.4. They are consistent with those obtained in ref. [19] by the dipole formula. There are only slight differences between the calculations without (solid) and with (dashed) the dipole approximation even for the $\Upsilon^{\prime}(3 S)$ case, whose radius is about $0.6 \mathrm{fm}$.

In conclusion, we have studied the diffractive photo- and leptoproduction of radially excited heavy quarkonia, and discussed the validity of the small-size color dipole approximation, which gives the smaller value of the $\psi^{\prime}$ to $J / \psi$ ratio compared with the experimental data. It is pointed out that the contribution from the higher order terms of $b^{2}$ in the $q \bar{q}-$ nucleon scattering amplitude, which is inherent in the original formula eq. (1), is important for the radially excited $\psi^{\prime}$ case. We have demonstrated that the calculation of eq. (1) without the dipole approximation provides a much larger value for the elastic photoproduction ratio $d \sigma\left(\psi^{\prime}(2 S)\right) / d \sigma(J / \psi(1 S))$ than the dipole result, and is consistent with the HERA data. As claimed in ref. [9], relativistic treatment of the charmonium system may be of some importance to construct the $\psi^{\prime}$ wave function. However, what we have shown here is that, even within our non-relativistic framework where there are some ambiguities of choosing the kinematics e.g. in eq. (3), substantial improvement for the $\psi^{\prime}$ production can be achieved by evaluating eq. (11) instead of eq. (5). Our results suggest that a careful treatment is necessary to study the radially excited light vector meson leptoproduction, e.g. $\rho^{\prime}(2 S), \phi^{\prime}, \cdots$ at moderate $Q^{2}$. We have also discussed the $\Upsilon^{\prime}(2 S, 3 S)$ production processes, and found that eq. (5) is a good approximation for the $b \bar{b}$ system because of its heavier quark mass.

\section{ACKNOWLEDGMENTS}

We thank Dr. D. Jido for useful discussions on the early stage of the present work. K.S. and A.H. are supported by JSPS Research Fellowships for Young Scientists. J.A. and T. H. 
are partially supported by JSPS grant No. 98360 . 


\section{REFERENCES}

[1] M. Ryskin, Z. Phys. C57, 89 (1993)

[2] S. Brodsky et al., Phys. Rev. D50, 3134 (1994)

[3] L. Frankfurt, W. Koepf and M. Strikman, Phys. Rev. D57, 512 (1998)

[4] M. Ryskin, R. Roberts, A. Martin, E. Levin, Z. Phys. C76, 231 (1997)

[5] A. Martin, M. Ryskin, T. Teubner, Phys. Rev. D55, 4329 (1997)

[6] J. Nemchik et al., JETP 86, 1054 (1998)

[7] A. Donnachie and P. V. Landshoff, Phys. Lett. B470, 243 (1999)

[8] M. Wüsthoff and A. Martin, J. Phys. G25, R309 (1999) and references therein

[9] P. Hoyer and S. Peigné, Phys. Rev. D61, 031501(R) (2000)

[10] C. Adolf et al. (H1 collaboration), Eur. Phys. J. C10, 373 (1999)

[11] J. R. Forshaw and D. A. Ross, "Quantum Chromodynamics and the Pomeron", (Cambridge University Press, London, 1997)

[12] W. Buchmüller and S.-H. Tye, Phys. Rev. D24, 132 (1981)

[13] C. Quigg and J.L. Rosner, Phys. Lett. B71, 153 (1977)

[14] A. Hayashigaki et al., in preparation

[15] E. Levin et al., Z. Phys. C74, 671 (1997)

[16] H.L. Lai et al., Phys. Rev. D55, 1280 (1997)

[17] We have also calculated eq. (11) using the 'linear' approximation [5, 15] for the unintegrated gluon density, instead of the approximation adopted in eq. (6). Resulting $\psi^{\prime}$ to $J / \psi$ ratio at $Q^{2}=0$ increases about $20 \%$ compared with the result shown in Fig.2, and comes closer to the data. More realistic approximation for $f\left(x, l_{t}\right)$ may improve our 
result. The result discussed in this paper should be considered as a lower bound of the ratio obtained by the calculation of eq. (1) without the dipole approximation.

[18] M. Glück, E. Reya and A. Vogt, Eur. Phys. J. C5, 461 (1998)

[19] L. Frankfurt, M. McDermott and M. Strikman, JHEP 9902, 002 (1999) 


\section{FIGURES}

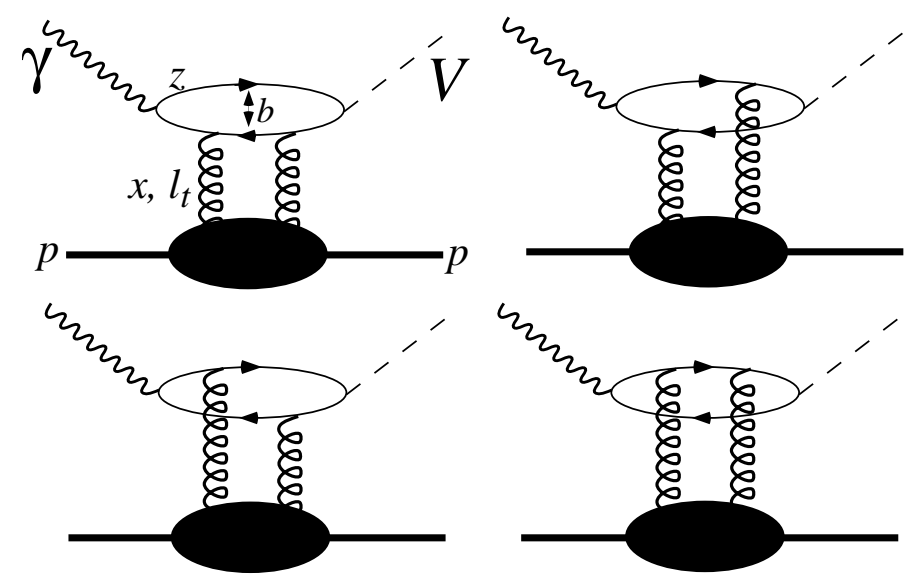

FIG. 1. Diagrams contributing to the diffractive vector meson production.

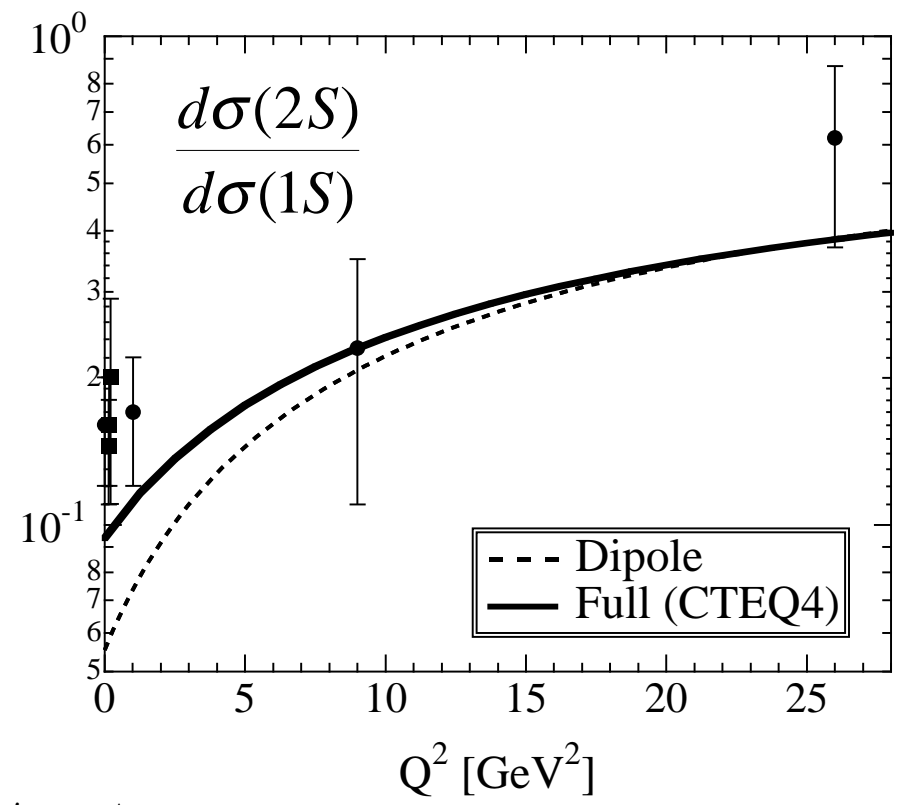

FIG. 2. Ratio of $\psi^{\prime}$ to $J / \psi$ production cross sections at $W=90 \mathrm{GeV}$. Results obtained by the calculations with $Q_{0}^{2}=0.8 \mathrm{GeV}^{2}$ and the color dipole approximation are depicted by the solid and dashed curves respectively. 


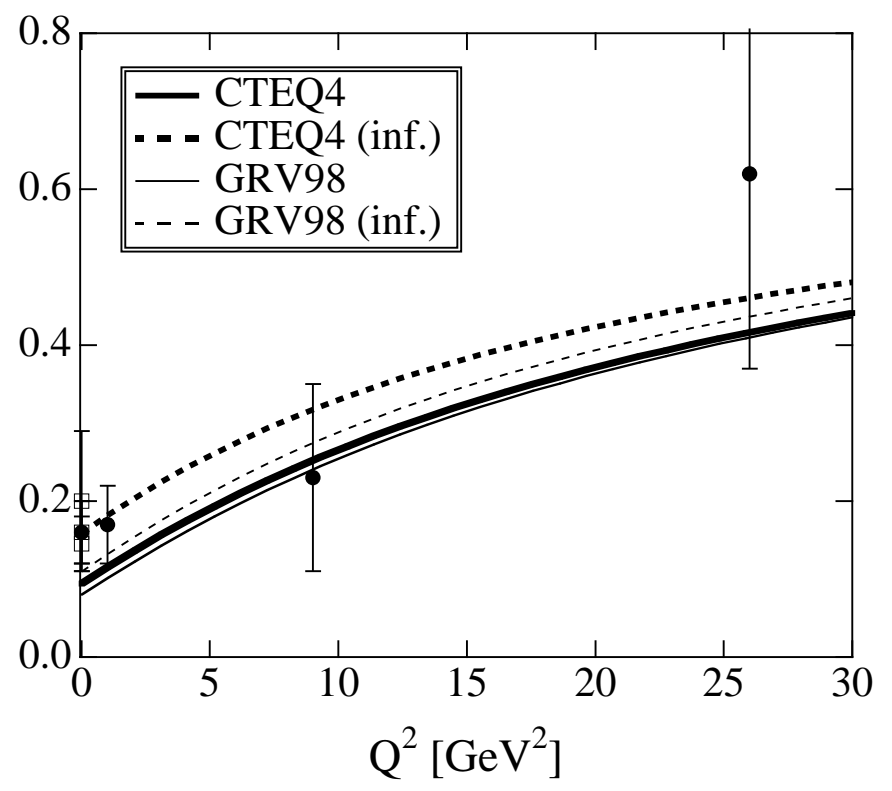

FIG. 3. $G\left(x, Q^{2}\right)$ and $Q_{e f f}^{2}$-dependence of the results with $Q_{0}^{2}=0.8 \mathrm{GeV}^{2}$. See text for details.

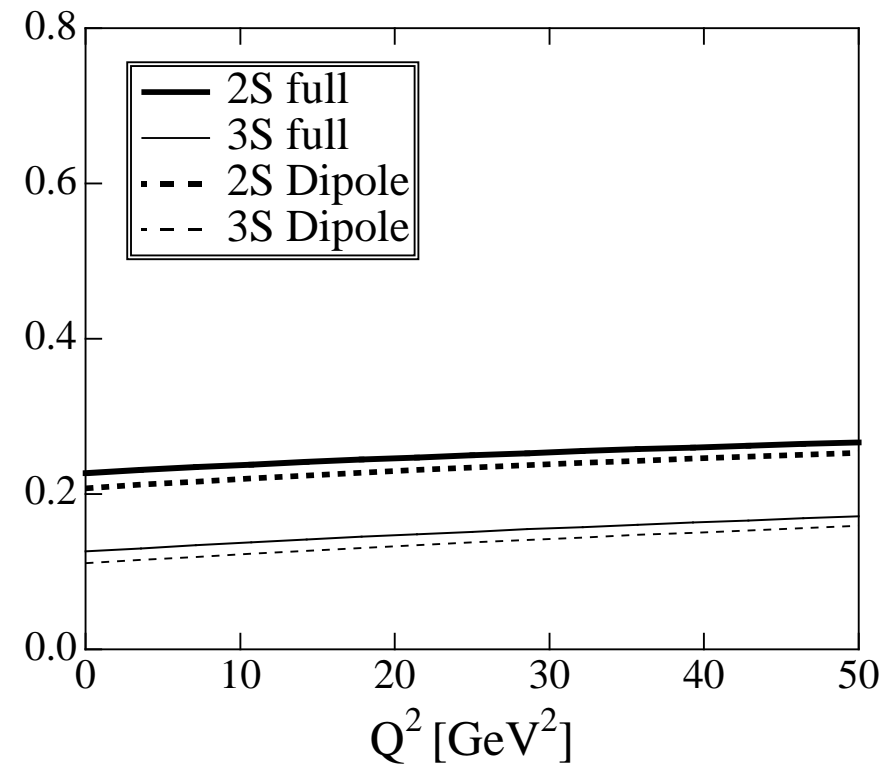

FIG. 4. Ratios of $\Upsilon^{\prime}(2 S, 3 S)$ to $\Upsilon$ cross sections. Thick solid and dashed curves denote $d \sigma\left(\Upsilon^{\prime}(2 S)\right) / d \sigma(\Upsilon(1 S))$ obtained by the full calculation and dipole approximation respectively. Thin solid and dashed curves indicate the corresponding results for $\Upsilon^{\prime}(3 S) / \Upsilon(1 S)$. 